\title{
PRESENTATION COMPLEXES WITH THE FIXED POINT PROPERTY
}

\author{
IVÁN SADOFSCHI COSTA
}

\begin{abstract}
We prove that there exists a compact two-dimensional polyhedron with the fixed point property and even Euler characteristic. This answers a question posed by R.H. Bing in 1969. We also settle another of Bing's questions.
\end{abstract}

\section{INTRODUCTION}

In his influential article "The elusive fixed point property" [3], R.H. Bing stated twelve questions. Since then eight of these questions have been answered [6]. In this paper we answer Questions 1 and 8.

Recall that a space $X$ is said to have the fixed point property if every map $f: X \rightarrow X$ has a fixed point. Motivated by an example of W. Lopez [8], Bing stated in [3] the following question.

Question 1.1 (Bing's Question 1). Is there a compact two-dimensional polyhedron with the fixed point property which has even Euler characteristic?

This question was studied in [10]. In [2] it was shown that such a space cannot have abelian fundamental group. In Corollary 2.4 we show that the answer to Question 1.1] is affirmative. Bing's Question 8 [3] may be rephrased as follows.

Question 1.2 (Bing's Question 8). What is the lowest dimension for a compact polyhedron $X$ with the fixed point property and such that a space $Y$ without the fixed point property can be obtained by attaching a disk $D$ to $X$ along an arc?

The answer to this question is clearly greater than 1. A one-dimensional polyhedron $X$ with the fixed point property is a tree, and then any space $Y$ obtained by attaching a disk along an arc is a contractible polyhedron. According to C.L. Hagopian [6], Bing conjectured that the answer to Question 1.2 is 2. This is the content of Theorem 2.8.

Acknowledgment: I am grateful to Jonathan Barmak, without his advice and suggestions this paper would not have been possible.

\section{BING GROUPS}

If $\mathcal{P}$ is a presentation, the presentation complex of $\mathcal{P}$ will be denoted by $X_{\mathcal{P}}$. Presentation complexes are in fact polyhedra. If a finite group $G$ is presented by a presentation $\mathcal{P}$ with $g$ generators and $r$ relators, then $r-g$ is at least the number of invariant factors of $H_{2}(G)$. If this lower bound is attained for $\mathcal{P}$, then the presentation is said to be efficient.

2010 Mathematics Subject Classification. 55M20, 57M20, 57M05.

Key words and phrases. Fixed point property, two-dimensional complexes, Schur multiplier. 
Definition 2.1. Let $G$ be a finite group and $d_{1}|\ldots| d_{k}$ be the invariant factors of $H_{2}(G)$. We say that $G$ is a Bing group if for every endomorphism $\phi: G \rightarrow G$ we have $\operatorname{tr}\left(H_{2}(\phi) \otimes \mathbb{1}_{\mathbb{Z}_{d_{1}}}\right) \neq-1$ in $\mathbb{Z}_{d_{1}}$.

Theorem 2.2. If $\mathcal{P}$ is an efficient presentation of a Bing group $G$ then $X_{\mathcal{P}}$ has the fixed point property.

Proof. Let $X=X_{\mathcal{P}}$ and $f: X \rightarrow X$ be a map. There is a $K(G, 1)$ space $Y$ with $X=Y^{2}$. Now $f$ extends to a map $\bar{f}: Y \rightarrow Y$. In the following commutative diagram, the horizontal arrows, induced by the inclusion $i: X \hookrightarrow Y$, are epimorphisms:

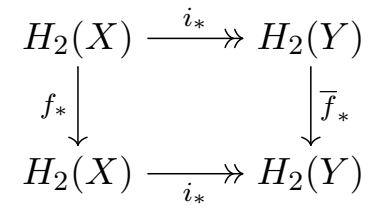

Let $d_{1}|\ldots| d_{k}$ be the invariant factors of $H_{2}(G)$. Since $\mathcal{P}$ is efficient, the rank of $H_{2}(X)$ equals the number of invariant factors of $H_{2}(Y)$. Therefore the horizontal arrows in the following commutative diagram are isomorphisms:

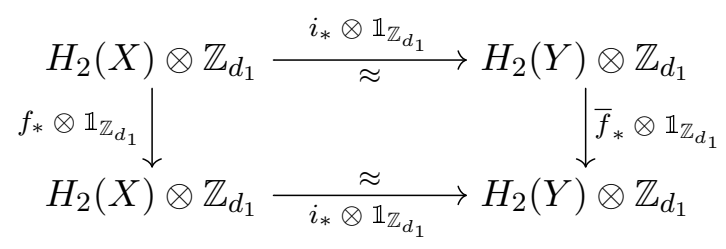

Now $\operatorname{tr}\left(f_{*} \otimes \mathbb{1}_{\mathbb{Z}_{d_{1}}}\right)=\operatorname{tr}\left(\bar{f}_{*} \otimes \mathbb{1}_{\mathbb{Z}_{d_{1}}}\right) \neq-1$ in $\mathbb{Z}_{d_{1}}$ since $G$ is a Bing group. Here we are using the natural isomorphism $H_{2}(B G) \approx H_{2}(G)$ of [9, Theorem 5.1.27]. Recall that every map $B G \rightarrow B G$ is induced, up to homotopy, by an endomorphism $G \rightarrow G$.

Finally we obtain $\operatorname{tr}\left(f_{*}\right) \neq-1$ in $\mathbb{Z}$, since tensoring with $\mathbb{Z}_{d_{1}}$ reduces the trace modulo $d_{1}$. So $L(f) \neq 0$ and, by the Lefschetz fixed point theorem, $f$ has a fixed point.

Proposition 2.3. The group $G$ presented by

$$
\mathcal{P}=\left\langle x, y \mid x^{3}, x y x^{-1} y x y^{-1} x^{-1} y^{-1}, x^{-1} y^{-4} x^{-1} y^{2} x^{-1} y^{-1}\right\rangle
$$

is a finite group of order 243. We have $H_{2}(G)=\mathbb{Z}_{3}$, so $\mathcal{P}$ is efficient. Moreover $G$ is a Bing group.

Proof. We will need the following GAP [5] program, that uses the packages HAP [4] and SONATA [1].

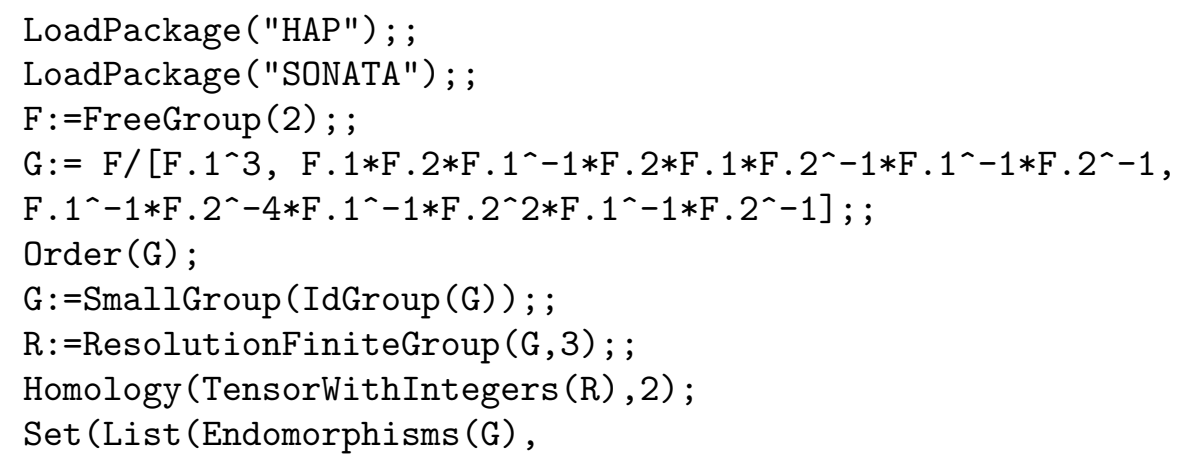


f->Homology (TensorWithIntegers (EquivariantChainMap (R,R,f)) ,2)));

The program prints the order of $G$, a list with the invariant factors of $H_{2}(G)$ and a list with the endomorphisms of $H_{2}(G)$ that are induced by an endomorphism of $G$. The output is:

[ 3 ]

$[$ [ f1 ] $\rightarrow[$ [identity ...> ], [f1 ] $\rightarrow[\mathrm{f} 1$ ] ]

Therefore $|G|=243$ and $H_{2}(G)=\mathbb{Z}_{3}$. Since for every endomorphism $\phi: G \rightarrow G$ we have that $H_{2}(\phi)$ is either the zero map or the identity, $G$ is a Bing group.

By Theorem 2.2 and Proposition 2.3 we have:

Corollary 2.4. The complex $X_{\mathcal{P}}$ associated to the presentation

$$
\mathcal{P}=\left\langle x, y \mid x^{3}, x y x^{-1} y x y^{-1} x^{-1} y^{-1}, x^{-1} y^{-4} x^{-1} y^{2} x^{-1} y^{-1}\right\rangle
$$

has the fixed point property. Moreover $\chi\left(X_{\mathcal{P}}\right)=2$.

Corollary 2.5. There are compact 2-dimensional polyhedra with the fixed point property and Euler characteristic equal to any positive integer $n$.

Proof. For $n=1$ this is immediate. For $n>1$ take a wedge of $n-1$ copies of the space $X_{\mathcal{P}}$ of Corollary 2.4 .

To prove Theorem 2.8 we will need another efficient Bing group:

Proposition 2.6. The group $H$ presented by $\mathcal{Q}=\left\langle x, y \mid x^{4}, y^{4},(x y)^{2},\left(x^{-1} y\right)^{2}\right\rangle$ is a finite group of order 16 . We have $H_{2}(H)=\mathbb{Z}_{2} \oplus \mathbb{Z}_{2}$, so $\mathcal{Q}$ is efficient. Moreover $H$ is a Bing group.

Proof. As above we will use a GAP program.

LoadPackage ("HAP") ; ;

LoadPackage ("SONATA"); ;

$\mathrm{F}:=$ FreeGroup (2); ;

$\mathrm{H}:=\mathrm{F} /\left[\mathrm{F} .1^{\wedge} 4, \mathrm{~F} .2^{\wedge} 4,(\mathrm{~F} .1 * \mathrm{~F} .2)^{\wedge} 2,\left(\mathrm{~F} .1^{\wedge}-1 * \mathrm{~F} .2\right)^{\wedge} 2\right] ;$;

$\operatorname{Order}(\mathrm{H})$;

$\mathrm{H}:=$ SmallGroup $(\operatorname{IdGroup}(\mathrm{H})) ;$;

$\mathrm{R}:=$ ResolutionFiniteGroup $(\mathrm{H}, 3)$; ;

Homology (TensorWithIntegers (R) ,2);

Set (List (Endomorphisms (H),

f->Homology (TensorWithIntegers (EquivariantChainMap $(R, R, f)$ ) , 2))) ;

The program produces the following output:

16

$[2,2]$

[ [ f1, f2 ] $\rightarrow$ [ <identity ...>, <identity ...> ],

$\left.[\mathrm{f} 1, \mathrm{f} 2] \rightarrow[\mathrm{f} 1, \mathrm{f} 2],[\mathrm{f} 1, \mathrm{f} 2] \rightarrow\left[\mathrm{f} 1^{\wedge}-1 * \mathrm{f} 2^{\wedge}-1, \mathrm{f} 2^{\wedge}-1\right]\right]$

This proves that $|H|=16, H_{2}(H)=\mathbb{Z}_{2} \oplus \mathbb{Z}_{2}$ and that $H$ is a Bing group.

We recall the following theorem: 
Theorem 2.7 (Jiang, [7, Theorem 7.1]). In the category of compact connected polyhedra without global separating points, the fixed point property is a homotopy type invariant.

Moreover, if $X \simeq Y$ are compact connected polyhedra such that $Y$ lacks the fixed point property and $X$ does not have global separating points, then $X$ lacks the fixed point property.

The following shows that the answer to Question 1.2 is 2:

Theorem 2.8. There is a compact 2-dimensional polyhedron $Y$ without the fixed point property and such that the polyhedron $X$, obtained from $Y$ by an elementary collapse of dimension 2 , has the fixed point property.

Proof. Let $\mathcal{P}$ and $\mathcal{Q}$ be the presentations of Propositions 2.3 and 2.6. By Theorem 2.2, $X_{\mathcal{P}}$ and $X_{\mathcal{Q}}$ have the fixed point property, so $X=X_{\mathcal{P}} \vee X_{\mathcal{Q}}$ also has the fixed point property. Since neither $X_{\mathcal{P}}$ nor $X_{\mathcal{Q}}$ have global separating points, by adding a 2-simplex, we can turn $X$ into a polyhedron $Y$, without global separating points and such that, by collapsing that 2-simplex, we obtain $X$. We have $H_{2}\left(\pi_{1}(Y)\right)=H_{2}\left(\pi_{1}\left(X_{\mathcal{P}}\right) * \pi_{1}\left(X_{\mathcal{Q}}\right)\right)=$ $H_{2}\left(\pi_{1}\left(X_{\mathcal{P}}\right)\right) \oplus H_{2}\left(\pi_{1}\left(X_{\mathcal{Q}}\right)\right)=\mathbb{Z}_{2} \oplus \mathbb{Z}_{6}$ and $\operatorname{rk}\left(H_{2}(Y)\right)=3$. By [2, Proposition 3.3] and Theorem 2.7, $Y$ does not have the fixed point property.

\section{REFERENCES}

[1] E. Aichinger, F. Binder, J. Ecker, P. Mayr, and C. Nbauer. SONATA - system of near-rings and their applications. GAP package, Version 2.6; 2012. (http://www.algebra.uni-linz.ac.at/Sonata/).

[2] J.A. Barmak, I. Sadofschi Costa. On a question of R.H. Bing concerning the fixed point property for two-dimensional polyhedra. arXiv:1412.8737

[3] R.H. Bing. The elusive fixed point property. Amer. Math. Monthly 76 (1969), 119-132.

[4] G. Ellis. HAP - Homological Algebra Programming. GAP package, Version 1.10.15; 2013. (http: //hamilton.nuigalway.ie/Hap/www/).

[5] The GAP Group. GAP - Groups, Algorithms, and Programming. Version 4.7.6; 2014. (http://www. gap-system.org).

[6] C.L. Hagopian. An update on the elusive fixed-point property. Open Problems in Topology. II, edited by E. Pearl, Elsevier B. V. 2007, 263-277.

[7] B. Jiang. On the least number of fixed points. American J. Math. 102 (1980), 749-763.

[8] W. Lopez. An example in the fixed point theory of polyhedra. Bull. Amer. Math. Soc. 73 (1967), 922-924.

[9] J. Rosenberg. Algebraic K-theory and its applications. Graduate Texts in Mathematics 147. SpringerVerlag, 1994.

[10] R. Waggoner. A method of combining fixed points. Proc. Amer. Math. Soc. 51 (1975), 191-197.

Departamento de Matemática, FCEyn-Universidad de Buenos Aires, Buenos Aires, ArGENTINA

E-mail address: ivansadofschi@gmail.com 